\title{
Finite Element Analysis on the Objective table of Virtual slice non-destructive measurement device
}

\author{
Yong Gan, Yang Chen, Jiaxing Li, Hanchao Wang \\ College of Mechanical \& Electrical Engineering, Guilin University of Electronic Technology, Guilin \\ 541004, China \\ 653194926@qq.com
}

Keywords: Objective table, Finite element modeling, Finite element analysis, Optimization schemes.

\begin{abstract}
For the minimum deformation requirement of an objective table in a new type of three-dimensional nondestructive measurement device measurement system, use the finite element analysis method to calculate the static response of the objective table under loading form, discussed the distribution characteristics of stress and strain of the objective table, put forward the optimization scheme. The results show that the optimized stress and strain of objective table meet the design's accuracy.
\end{abstract}

\section{Introduction}

The application of nondestructive measurement is becoming more and more extensive in the nowadays. But the measurement for the inner contour of work piece is still not yet completely mature, and all the countries are devoting to the research of this field. In this case, the design and development of the three-dimensional nondestructive measurement device, which based on the virtual slides technique, and its corresponding measurement system, will has the prominent contribution in three-dimensional nondestructive measurement for our country [1]. Wherein, the precise measurement system is the key point of the three-dimensional nondestructive measurement techniques. The design of the objective table is the core of the measuring system. Therefore, the design of the objective table has a great influence on the measurement accuracy [2].

With the maturing of the finite element technology, especially the development of the finite element software, the finite element analysis has been widely used in the engineering application [3]. In this paper, through establishing the finite element analysis model of the objective table, the structural optimization design for the objective table is carried out by combining the finite element analysis method and the optimization method, and the results will be compared with the before optimization.

\section{The finite element analysis model of Objective table}

Create geometric model of objective table. According to a requirement of design, the geometry model of objective table is built by the software SOLIDWORKS, as shown in figure 1

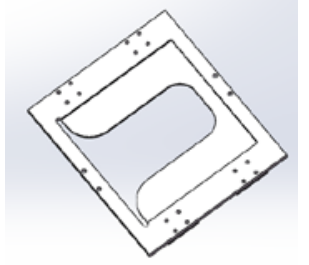

Figure 1 the geometry model of objective table by the software SOLIDWORKS

Define the material properties. For finite element model, the material properties of the model need to be defined [4]. The raw material of the objective table is hard aluminum alloy. It is need to input the material properties of the material in the ANSYS. For the normal static analysis, define the material properties, as shown in table 1. 
Table 1 objective table of material properties

\begin{tabular}{|c|c|c|c|c|}
\hline Material & $\begin{array}{c}\text { Density } \\
(\mathrm{g} / \mathrm{cm} 3)\end{array}$ & $\begin{array}{c}\text { Modulus of elasticity } \\
(\mathrm{E} / \mathrm{GPa})\end{array}$ & Poisson's ratio (u) & $\begin{array}{c}\text { yield strength } \\
\text { (MPa) }\end{array}$ \\
\hline LY12 & 2.7 & 70 & 0.31 & 290 \\
\hline
\end{tabular}

Unit selection and meshing. Choose the automatic meshing. ANSYS 's mesh automatic classification is based on the shape of the object to be tested to analyze its main stress parts of discretion, its meshing model is shown in Figure 2. First of all, because the objective table is connect with the four mobile sliding blocks, four connections are in an area of concentrated stress; Second, due to boundary effect the bearing surface boundary is also the internal force concentrated area; Besides, according to the principle of moment concentration the middle position of the bearing surface is one of internal force concentrated area. So the mesh in this three regions of bearing surface is quite tense [5]

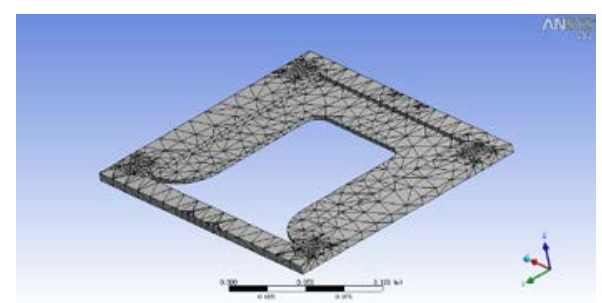

Figure 2 Objective table meshing model's diagram

Imposing load in objective table. According to the objective table design in the measuring apparatus, the objective table uses four moving slider as a fixed support. While applying the constraint on the table, the contact surface and bolt positioning holes are fixed as restraints. Then for the assembly of the positioning clamping mechanism, the four faces of the receiving groove in the $\mathrm{Y}$ direction with the check constraint are formed. Table's load is applied to the bearing surface, and according to this thesis which limit the measurement range of virtual slice non-destructive measurement device, it means that load capacity is limited between $0 \mathrm{~N}$ and $200 \mathrm{~N}$, namely $0 \sim 20 \mathrm{~kg}$ [6].

Strength analysis of objective table. After finishing the setting in ANSYS, the stress-strain diagram is achieved by solving. Figure 3 and 4 are respectively the total stress-strain diagram of the objective table in maximum load $200 \mathrm{~N}$.

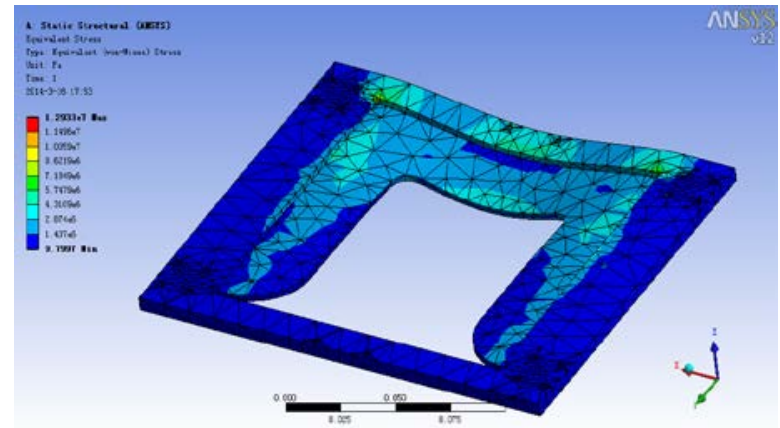

Figure 3 object stage stress diagram

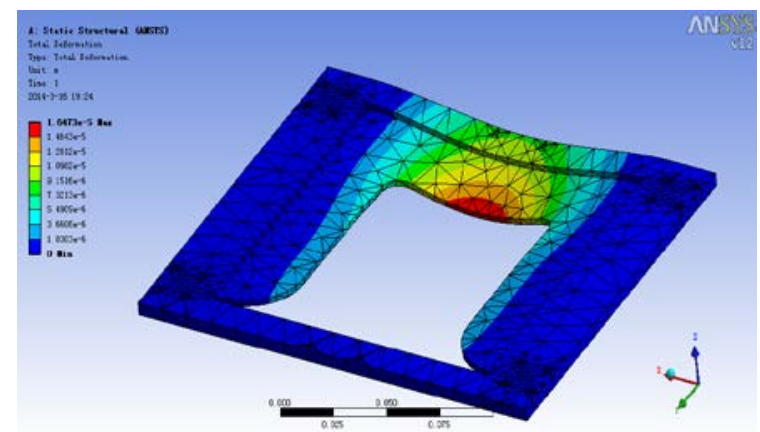

Figure 4 object stage strain diagram

Loading units should seek to show that stress is concentrated in three areas: slider fixed contact, bearing surface boundary area and hosted call center line area and analysis, which are in accordance with analysis described above. Combined with the strain as shown in the figure, contingency with the u-shaped Groove to weaken, which strain the most concentrated area in hosted call center line with a U-shaped trough around the region, it is clear that the region is the maximum deformation of the bearing surface area. Aiming at above phenomenon, first of all, by collecting the area strain stress curves of impact are corresponding laws, as a basis for structural optimization. We can get the area of 
the relationship between strain and load curve when the loading between $0 \sim 200 \mathrm{~N}$ and the strain values are taken every $10 \mathrm{~N}$, as shown in figure 5.

The figure 4 and 5 illustrates that the relation between the strongest strain area's strain values of objective table and load is a linear change as a whole. When the maximum load is $200 \mathrm{~N}$, the maximum strain value is $2.52 * 10-5$, and total deformation is $0.016 \mathrm{~mm}$.

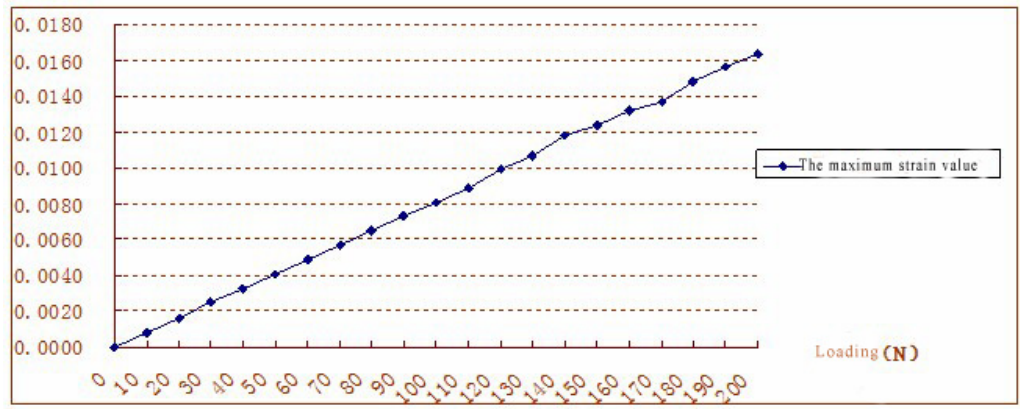

Figure 5 strain relationship with load

\section{The optimal design}

Based on finite element analysis, the largest total deformation of the object stage is $0.016 \mathrm{~mm}$, and the accuracy requirement of structure design is $0.01 \mathrm{~mm}$ [7-8]. Therefore it failed to meet the design requirements, and need to optimize the design. Because the total deformation is the accumulation of each strain, the large area of the total deformation is not the most concentrated stress area, as shown in Figure 3 and Figure 4. So in the structure something can be adjusted, such as enlarging the strain concentration area, reducing the area of lesser stress. Thus reducing the maximum of the total deformation, strain area is reduced.

According to the above analysis, the objective table structure optimization model and the grid are shown in figure 6 . The bearing surface area of the two side of the optimized objective table's u-shaped slot is increased from the original $150 \mathrm{~mm} \times 30 \mathrm{~mm}$ to $150 \mathrm{~mm} \times 40 \mathrm{~mm}$. The bearing surface area of the U-shaped groove bottom is reduced from the original $30 \mathrm{~mm}$ to $20 \mathrm{~mm} \times 150 \mathrm{~mm}$ $\mathrm{x} 150 \mathrm{~mm}$., and the bottom excessive radius is extended to $20 \mathrm{~mm}$. To do the static analysis, the stress and total deformation diagram are obtained, as shown in Figure 7 and Figure 8.

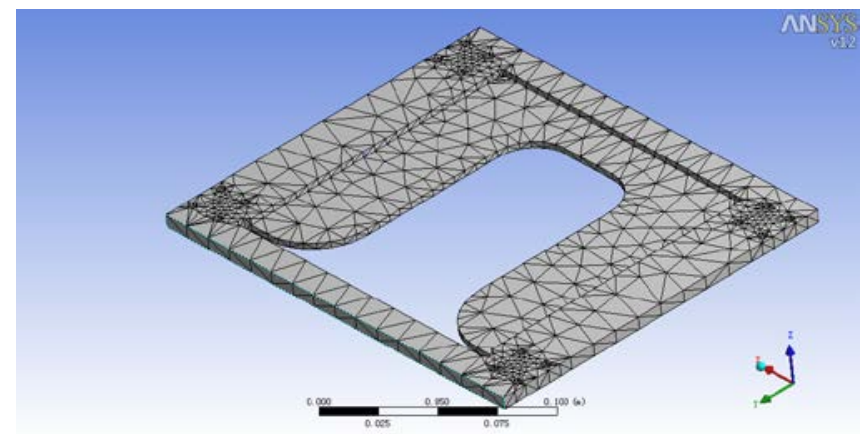

Figure 6 the optimized objective table grid model

Compared figure 4 with figure 7 , stress figure of the Before and after optimization stable show that stress of the optimized stable are mostly found in the two sides of bearing surface's U-shaped slot, which reduced the stress concentration phenomenon of the symmetry center line. In addition, from the whole distortion pattern's show of the optimized stable, its strain area are more evenly than the before optimization, and its maximum total distortion reduced from $0.016 \mathrm{~mm}$ to $0.012 \mathrm{~mm}$. To counter this phenomenon, the strain's finite element analysis of reducing the size of u-shaped slot at the bottom of the bearing surface are carried out, and the Bearing surface's size and the total distortion curve are obtained, as shown in figure 9. 
Graph shows that when the size reached to $10 \mathrm{~mm}$, the total maximum distortion value of the Objective table is the minimum. Therefore, the optimized u-shaped slot's size is $70 \mathrm{~mm} * 140 \mathrm{~mm}$. At this time, the total maximum distortion is $0.008 \mathrm{~mm}$.

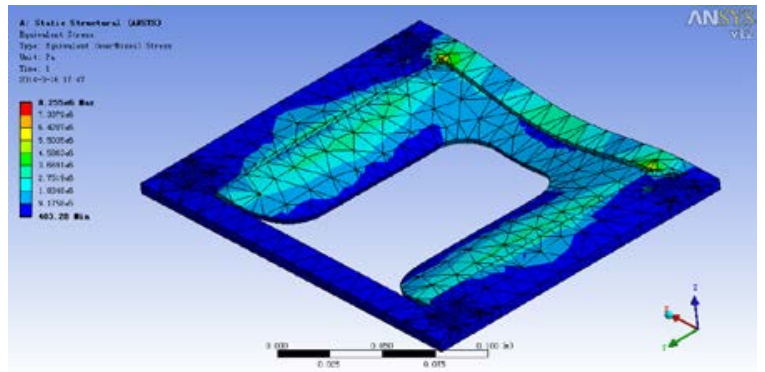

Figure 7 optimized object stage total stress diagram

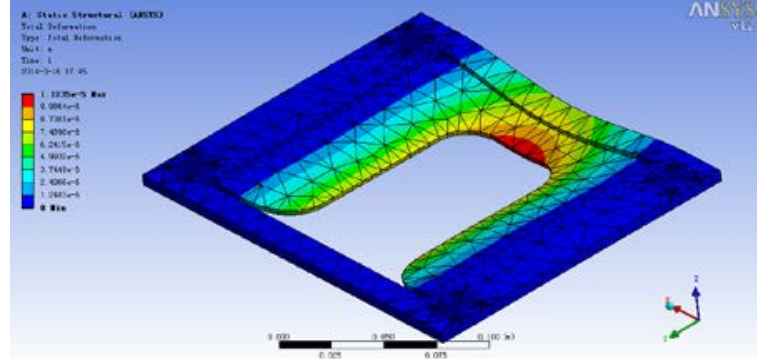

Figure 8 optimized object stage total deformation figure

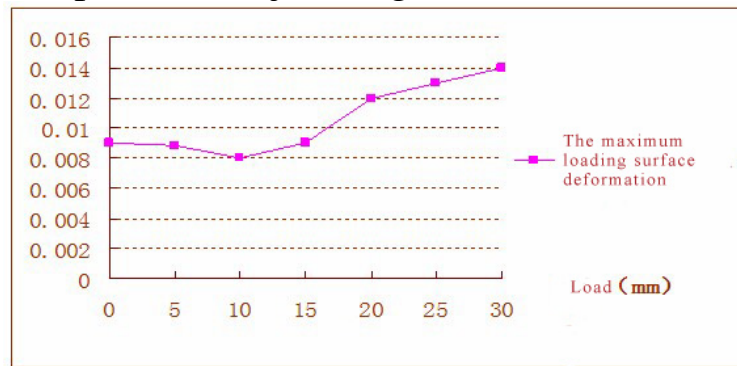

Figure 9 The overall size and the maximum loading surface deformation curve

\section{Summary}

Under the working load, finite element calculation of structure strength which is the Objective table of Virtual slice non-destructive measurement device is done by using the ANSYS software, and the results are discussed, and finally the structure is optimized. The results show that the optimization method can effectively reduce the deformation of the object table and precision meet the design precision requirements.

\section{Acknowledgements}

This project is financially supported by the National Natural Science Foundation of China (51265007).

\section{References}

[1] Gan Yong. China Mechanical Engineering.2012, 25(10):1350-1353 In china.

[2] Gan Yong. China Machinery Design \& Manufacture.2014.6:140-142 In china.

[3] Gong Shuguang, Xie Guilan. China Machine Design and Research. 2004,20(1).83-85 In china.

[4] Jiang Libiao. China Machinery Design \& Manufacture. 2014(1) :183-185 In china.

[5] Li Yugang. Mechanical Structure Optimization and Research of Nondestructive Measurement Device on Virtual Slide [D]. Guilin Guilin University of Electronic and Technology.

[6] Gan Yong, Sun Ning. China Journal of Machine Design,2008,25(10) :8-11 In china. 
[7] Gan Yong, Sun Ao-ran, Sun Ning, Wei Lipu. China Manufacturing Automation, 2011, 33 (9):48-50) In china.

[8] Gan Yong, Li Yugang, Zhang Fu-zhi. Applied Mechanics and Materials, 2013:511-514. 